\title{
A Qualitative Case Study Exploring the Experiences of Caregivers Using a Patient Navigation Centre for Children with Complex Care Needs
}

\author{
Rosalinda Oi-Lin Knight' ${ }^{1}, \mathrm{MD}$; Alison Luke², PhD; and Shelley Doucet ${ }^{2,3}$, PhD, \\ RN \\ ${ }^{1}$ Department of Family Medicine, Dalhousie University \\ 2Department of Nursing and Health Sciences, University of New Brunswick \\ 3Dalhousie Medicine New Brunswick
}

DOI: https://doi.org/10.15273/hpj.v1i2.10653

\begin{abstract}
Introduction: Approximately 15\% of children in North America live with a complex health condition. When caring for these children, it can be difficult and time-consuming to determine what services are available to meet their complex needs. Method: The aim of this research project was to explore the experiences of families using NaviCare/SoinsNavi, a patient navigation centre for children/youth with complex care needs and their families. Six families were assessed longitudinally over a six- to eight-month period. Semi-structured interviews were done at intake, three to five months, and six to eight months. Families also kept a monthly diary recording their experiences. Results: Families were satisfied with the services provided by NaviCare/SoinsNavi. They found it comforting to have someone that would take the time to listen to their concerns and was able to provide recommendations for services. Discussion: This longitudinal case study shows the benefit of navigation centres for children/youth with complex care needs and their families.
\end{abstract}


A Qualitative Case Study Exploring the Experiences of Caregivers Using a Patient Navigation Centre for Children with Complex Care Needs

\section{Introduction}

Approximately $15 \%$ of children in North America experience a chronic health condition (Kaufman et al., 2007). These children often have unique health-care experiences that can make navigating the health care system challenging. They often require multiple visits to specialists and may require hospitalizations (Colver et al., 2013). While there is no single definition for a child with complex care needs, for the purpose of this study, this refers to a child or youth with "multidimensional health and social care needs in the presence of a recognized medical condition or where there is no unifying diagnosis..." (Brenner et al., 2018, p. 1647). These children are significant users of the health care system and related services, requiring a coordinated effort by the family as well as the care team.

The first patient navigation program was developed in Harlem, New York, by Dr. Harold Freeman in 1990 (Freeman \& Rodriguez, 2011). Since that time, patient navigation has increased in use across North America. The aim of patient navigation is to guide and orient patients through the health care system, connecting patients' unmet needs to appropriate resources to decrease fragmentation, improve access, and support the coordination of care (Fillion et al., 2012; Freeman \& Rodriguez, 2011; McMullen, 2013). While the first patient navigation centres focused on cancer patients (Freeman \& Rodriguez, 2011), it is becoming evident that patient navigation programs may be useful for other medically underserved populations, such as those who live in rural areas, due to the limited resources in these communities (Paskett et al., 2011; Weinhold \& Gurtner, 2014). A recent environmental scan identified 19 pediatric patient navigator models in Canada (Luke et al., 2020). Patient navigation is an area of research that is increasingly shown to improve outcomes for children with complex care needs (American
Academy of Pediatrics Council on Children with Disabilities, 2005). However, studies exploring the experiences of participants using navigating services is limited. Navigating services and resources to meet the complex care needs of children can be especially difficult for families and even the care team (Charlton et al., 2017). Based on a needs assessment completed prior to 2017 with input from various stakeholders (Doucet et al., 2017), it was determined that there was a need for a patient navigation centre for children and youth with complex care needs and their families in New Brunswick, Canada (Charlton et al., 2017). As such, NaviCare/SoinsNavi (NaviCare hereafter) was launched in January of 2017 (Doucet et al., 2019) with the following goals: (a) to ensure the integration of health, education, and social services for children and youth with complex care needs; (b) to advocate for children and youth with complex care needs and their families; and (c) to offer families a setting to voice their needs and mentor/support other families.

NaviCare is a free virtual research-based service that is available primarily through phone or email for children and youth (25 years of age or under), their family, and the care team. It is currently housed at the University of New Brunswick in Saint John. Families are connected to a patient navigator (PN) who is either a Registered Nurse or lay navigator. The ultimate goal of the PN is to coordinate access to services and resources and act as an integral part of the care team, helping children and youth and their families with their health-care journey. In the literature, there is limited research evaluating patient navigation, especially when dealing with navigation centres whose mandate involves children with complex care needs and their families.

\section{Objective}

Patient navigation has been increasingly used to help integrate care since the 1990s and has expanded to include the pediatric population. There are few studies that highlight the experiences of caregivers who use these services. The aim of this research project was to 
explore the experiences of caregivers of children and youth with complex care needs using NaviCare over three time points. This project is part of a larger program evaluation being undertaken to study the impact of NaviCare. Please see Luke et al. (2020) for additional details on the larger program evaluation.

\section{Methods}

\section{Design}

A longitudinal qualitative descriptive case study design (Yin, 2014, pp. 5-6) was used to explore the experiences of caregivers of families using NaviCare over three time points. Case studies allow the researcher to conduct an in-depth exploration of a subject over time.

\section{Sample}

Participants were recruited to participate in the study when they first accessed the NaviCare service. Inclusion criteria included (a) being a client of the NaviCare services, and (b) were seeking navigational support for a child with a high level of complexity, being thus more likely to use the centre over an extended period. There were no exclusion criteria. For this study, "long-term" was defined as six months or more. In total, ten potential participants were identified by the PN. Their files were reviewed by the research leads, who confirmed whether each participant met the study inclusion criteria. The primary researcher called potential participants by phone and invited them to participate. If the family agreed, an initial interview was scheduled. If the families declined to participate, they did not have to provide an explanation why.

\section{Ethics}

This project received ethics approval from the University of New Brunswick in Saint John (REB \# 026-2016). The PN obtained verbal consent from the families on their willingness to be contacted by a member of the research team at their initial intake with NaviCare. Written consent to participate in the study was obtained at the beginning of the initial interview. Participants were given $\$ 100$ in gift cards to a local grocery store for their participation. These gift cards were given as a $\$ 25$ gift card at the first and second interview and a $\$ 50$ gift card at the third interview. There were cases where additional family members, who did not give consent to be interviewed, were present and accidentally recorded at the time of the interview. In these circumstances, their comments were omitted and not transcribed. All recordings were deleted immediately following transcription.

\section{Data Collection: Interviews and Diaries}

Two data collection methods were used to gain insight into the experiences of caregivers of families. These included traditional semistructured interviews and the use of diaries to augment data collected from interviews.

Interviews were all conducted in person. Families were given the option to be interviewed in their home or another location of their choice. Participants were interviewed between 2017 and 2019. Participants were given the option to be interviewed in English or French. All participants opted to be interviewed in English, and translational services were not required.

Semi-structured interviews were conducted at three points in time: intake, three to five months, and six to eight months. The same interview guide was used for all three interviews. Interview questions were designed with open-ended questions to allow participants to fully express their experiences and guide the interview. Interview questions were divided into five categories: (a) Satisfaction/experience with NaviCare; (b) Knowledge gained from accessing NaviCare; (c) Experiences with coordination, integration, and continuity of care; (d) Changes in quality of life; and (e) Suggestions for improvement of the centre. Interviews were recorded, and each lasted approximately 20 minutes.

At intake, participants were also asked to keep a monthly diary to record their experiences. Participants were provided with an 
iPod touch and paper diary and were given the option to record their entries using an audio, video or written diary format. A diary guide was provided in both English and French to assist participants; however, they were informed that the content of the diaries could be creative to reflect their own experiences with NaviCare. The diary guide prompted participants to document both positive and negative experiences using NaviCare. The use of diaries allows experiences to be recorded in close temporal proximity to their occurrence, which contrasts with more traditional qualitative methods, such as semi-structured interviews, in which there may be a span of time between an experience and the recording of that experience (Bolger et al., 2003).

\section{Data Analysis}

Interviews and diary data were transcribed verbatim. Transcription of both the interviews and diaries were done solely by the principal researcher. Transcripts were read over by the primary researcher for accuracy. Transcripts were de-identified, and participants were assigned a code. Both interview and diary data were analyzed together with the intention that diary data would enrich the interview data. The lead author used Microsoft Word with the highlight function to extract codes and identify themes. The lead author met with co-authors to review codes and themes. The analysis was guided by Braun and Clarke's six phases for thematic analysis (Braun \& Clarke, 2006), which are the following:
1. familiarization with the data,

2. generation of initial codes,

3. search for themes,

4. review of themes,

5. definition and naming of themes

6. generation of a report.

\section{Results}

In total, ten families were identified by the PN and subsequently contacted by the research team. Four families were not included in the study: three of these families declined to participate, while one other family was not deemed to be appropriate as they did not anticipate using the centre long-term (over 6 months). Families that declined did not provide a reason as to why they did not want to participate in the study. Six families were initially enrolled in the case study. Their demographic information is included in Table 1. Four of these families completed the case study, while two others completed one interview but withdrew afterwards (Figure 1). From the six families initially enrolled, five participants were mothers, and one was a grandmother. All participants were the child/youth's primary caregiver. Data from withdrawn participants was included in the study with their consent. All interviews were conducted in the participant's home as preferred by the participant. All participants opted to be interviewed in English.

One of the final four participants did not complete the diary entries. This participant informed the research lead at the final interview and diary collection that they had lost their

\section{Table 1}

Demographic Information from Participants

\begin{tabular}{|l|l|l|l|l|l|}
\hline $\begin{array}{l}\text { Participant } \\
\text { Number }\end{array}$ & $\begin{array}{l}\text { Relationship to } \\
\text { Child }\end{array}$ & $\begin{array}{l}\text { Primary Language } \\
\text { Spoken }\end{array}$ & $\begin{array}{l}\text { Condition of } \\
\text { Child }\end{array}$ & $\begin{array}{l}\text { Age of } \\
\text { Child }\end{array}$ & $\begin{array}{l}\text { Sex of } \\
\text { Child }\end{array}$ \\
\hline 3 & Grandmother & French & Neurological & 8 & M \\
\hline 5 & Mother & English & Mental Health & 10 & M \\
\hline 6 & Mother & English & No Diagnosis & 8 & M \\
\hline 8 & Mother & English & Cardiac & 16 & M \\
\hline 9 & Mother & English & Gastrointestinal & 3 & M \\
\hline 10 & Mother & English & Neurological & 9 & F \\
\hline
\end{tabular}




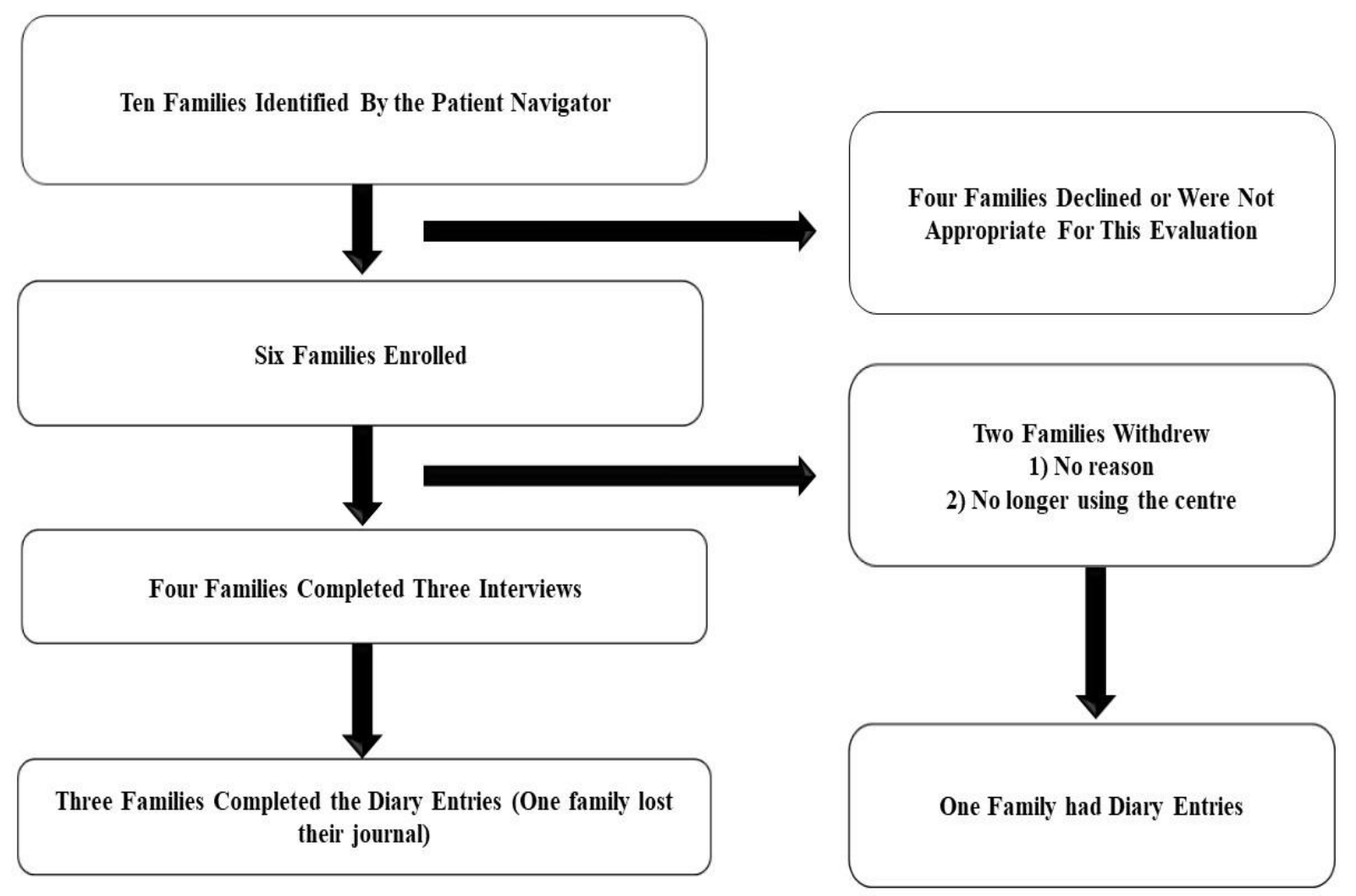

Figure 1

Outline of Patient Enrollment in Study

Note. An outline of patient enrollment, including the number of families that completed the three semi-structured interviews and diary entries.

diary. They were not excluded from the study and their interview data was analyzed. One family that withdrew partway through the study completed four diary entries. Three participants preferred to use written diaries, while one used the iPod for audio diary entries. Table 2 outlines details of the diary entries.

\section{Themes Emerging from Interviews and Diaries}

Based on the transcripts, codes were used to determine themes from both interview and diary data. In general, data emerging from diaries coincided with interview data. In addition, diary data provided additional details about programs and services suggested by the PN that participants could not remember at the time of the interview. There were five main themes that emerged from the data: (a) a strong sense of support, (b) sharing knowledge, (c) improved communication with the care team, (d) quality of life and (e) suggestions to improve the navigation centre.

\section{Theme 1: A Strong Sense of Support}

All participants described being satisfied with the services provided by NaviCare. Participants felt that the PN was a strong pillar of support. They regularly described situations where they felt overwhelmed and found that the PN provided the emotional support required to care for their child.

... the [PN] kept me going while everything was really stressful, they were very supportive. And having two children with special needs, one with extreme 
special needs is difficult. And when you are fighting a battle to get help and care knowing that I had two people that I could call at any time that, yeah that made the difference... (Participant 5)

Multiple caregivers of families described feeling alone and not heard by the system. In these situations, participants found that NaviCare was able to provide support by carefully listening to parents and what they had to say.

Especially people who have chronically ill children and you don't have a diagnosis it becomes extremely frustrating and you feel alone. Like sometimes you feel like oh yeah no no they are listening but they aren't hearing what you are saying [...] you know that validation that it's okay, we are going to get to the bottom of this and I'm going to help you get there. (Participant 9)

In addition to support from the PN, some participants also mentioned that they felt they would benefit from the creation of a support group for parents who have children with complex care needs.

\section{Theme 2: Sharing Knowledge}

In general, caregivers of families were happy with the PN's knowledge of available programs and services. The PN was found to have provided recommendations for a large variety of programs and services. Different caregivers requested different services based on their needs. As an example, some participants felt very comfortable navigating the health care system but struggled to navigate the government programs or the school system. In these cases, the PN provided information about tax reimbursements, the Department of Social Development, forms for schools and letters of support. Other caregivers felt as though they struggled with accessing health-care services locally. In these cases, the PN helped by contacting pediatricians and informing parents about services such as respite care, dietetics and multidisciplinary teams. One family developed a strong working relationship with the PN where they would share information and inform the PN about services and programs that they may not have been aware of. As one participant stated, "So, they kinda learned some stuff from me and I learned some stuff from them. So, it was kinda a teachable moment for both of us" (Participant 5).

During their second interview, one participant described finding a program themselves and wishing that NaviCare had directed them there earlier. This is discussed further below under the theme "Suggestions to improve the navigation centre". Participants also commented on learning about programs from other services that they were originally directed to by NaviCare. For example, the PN connected one family with a social worker, who then referred them to additional resources. As stated by this participant, "without one it wouldn't have led to the other" (Participant 10).

Table 2

Details of the Diary Entries

\begin{tabular}{|l|l|l|l|l|}
\hline Participant & $\begin{array}{l}\text { Number of } \\
\text { Interviews }\end{array}$ & $\begin{array}{l}\text { Number of } \\
\text { Diary Entries }\end{array}$ & Type of Diary & $\begin{array}{l}\text { Avg. Length of Diary } \\
\text { Entry }\end{array}$ \\
\hline 3 & 3 & 0 & N/A & N/A \\
\hline 5 & 3 & 8 & Written & 25 words \\
\hline 6 & 1 & 0 & N/A & N/A \\
\hline 8 & 1 & 4 & Audio & 1 min 30 sec \\
\hline 9 & 3 & 7 & Written & 206 words \\
\hline 10 & 3 & 6 & Written & 43 words \\
\hline
\end{tabular}


Theme 3: Improved Communication with the Care Team

Participants felt as though communication with the care team was improved. The PN communicated with various members of the care team, in some cases expediting appointments with pediatricians and other health-care providers. As stated by one participant, "I found that it really helped me communicate with the other people looking after [Son]" (Participant 9).

Parents found that having the PN advocate and communicate on their behalf made other health-care providers pay more attention to their concerns. Participants also found that the PN helped them better prepare for visits with health-care professionals, advising them to make a list of questions they should ask at these appointments.

\section{Theme 4: Mixed Responses Concerning Quality of Life}

Some participants describe an improvement in the baseline of their quality of life after contacting NaviCare. They found that the PN helped decrease the amount of work they had to do. One participant commented that "[the PN] has been able to take some off of my plate for me ... so it kind of lessens the load" (Participant 5).

While none of the participants described having their quality of life decrease after calling NaviCare, some participants describe no change in baseline of their quality of life: "My quality of life, no I would say it's the same. It's the same, yeah" (Participant 3).

\section{Theme 5: Suggestions to Improve the Navigation Centre}

Participants were asked what suggestions they had to improve NaviCare. While most participants were happy with the services provided, one participant believed that NaviCare should increase the repertoire of services recommended by the PN: "We discovered there is a camp for children and adults living with disabilities. I think this is something that should be added to the services NaviCare help with" (Participant 10).
Based on factors beyond the control of the centre, there was a switch in the PN during the time period in which this longitudinal study took place. Two participants commented on how there was a period where they were not contacted, which left them wondering if the service was still running. One of these participants commented on how part of what they loved about NaviCare was that they did not have to repeat their story multiple times. Having this switch was quite difficult for them, as they relied heavily on the prior PN for support and were wary about becoming attached to a new PN. This difficulty was reflected in one client's diary and had an impact on their experience with the centre.

Another suggestion brought to light by participants was the need for an increase in marketing and promotion for the centre. Participants found that other parents in their social circles who they believe would benefit from NaviCare were unaware of the service. Additionally, participants found that they would have to explain what NaviCare was to healthcare professionals. Finally, participants found that they would like to have a face-to-face meeting with the PN to see who the person was on the other end of the phone.

\section{Discussion}

The aim of this research project was to explore the experiences of caregivers of children and youth with complex care needs using NaviCare over three time points. There were five main themes that emerged from the data: (a) a strong sense of support, (b) sharing knowledge, (c) improved communication with the care team, (d) quality of life and (e) suggestions to improve the navigation centre.

All participants were satisfied with the services provided by NaviCare. The most recurring theme among all participants was that they found NaviCare to be a pillar of support. Prior to contacting the centre, participants felt alone and not taken seriously by the health care system. NaviCare was a service that helped validate their concerns and helped them through tough situations by providing 
emotional support. Participants felt as though they would benefit from a support group for parents with children with complex care needs. This is consistent with a scoping review done by King et al. (2017) on family-oriented services in pediatric rehabilitation in which caregivers identified the need for emotional support. Other studies have highlighted that being a caregiver of children with complex care needs can have an impact on emotional and mental health (Caicedo, 2014; Ellenwood \& Jenkins, 2007). It appears that NaviCare is filling that role of a support group for parents by providing an environment where parents were able to express their concerns to someone who has the time to address and validate them. This mirrors other programs that use PNs. As an example, a study by Gotlib Conn et al. (2016), which followed a patient navigation service for cancer patients, demonstrated that PNs provided holistic support, including emotional support to participants and their families.

In general, participants were satisfied with the number of services and resources recommended by the $\mathrm{PN}$ and by the improvement in the coordination of their child's care as a result of the PN bringing the team together. This is in keeping with not only the original aims of the centre, but also with other patient navigation programs whose outcomes include increased communication with services and care providers and increased patient satisfaction regarding services (Valaitis et al., 2017). The theme "sharing knowledge" also highlighted the back-and-forth relationship that caregivers have with the PN. Participant 5 was quoted saying "So, they kinda learned some stuff from me and I learned some stuff from them. So, it was kinda a teachable moment for both of us." The caregivers themselves are also a wealth of knowledge.

Interestingly, while most participants were satisfied with the services, the effects on quality of life were mixed among participants. Participants whose quality of life was unchanged involved cases that were not yet resolved (i.e., they remain clients of the centre). As an example, one participant interviewed in the summer months was scheduled to start receiving respite care for their child in the school year. This participant postulated that their quality of life would improve once they had a care worker who could start coming during the school year. In contrast, participants whose quality of life had improved had found services that helped them cope with their child's complex care needs. In the literature, the effects of patient navigation on quality of life are mixed (Paskett et al., 2011). However, a more recent study by King et al., (2017) suggested that PN services can help lessen the burden of caregivers and improve the caregiver experience. Given that these children have complex care needs, longer term studies will likely be required to determine if there is a trend of improvement in quality of life.

\section{Limitations}

One limitation of this study is the small sample size. Moreover, given that the PN flagged participants who might be long-term cases with NaviCare, this potentially introduced bias as only flagged participants were contacted for enrollment in the study. The participants were required to self-report satisfaction and experience, which is dependent on participants' recall and recollection. Although participants were informed that only the research team had access to the findings, the information shared may have been limited, as participants may have not wanted to give negative feedback for fear that it may get back to the PN. An additional limitation is that the centre was in the pilot stages when the data for this study was collected. Waiting until the early kinks were ironed out may have led to differences in the reported experiences. Finally, the average interview time was short, at approximately 20 minutes, which may have been too little time to generate answers.

\section{Recommendations for Navigation Centres}

Our recommendations for patient navigation programs are based on the suggestions from participants of this qualitative study. The first recommendation is to ensure 
proper marketing of a new navigational centre. Participants found it difficult having to explain what NaviCare was to other parents, organizations, and health-care providers. They suggested increased marketing and promotion of the centre to make it more well known. Our suggestion for this would be to present the centre at Grand Rounds teaching events at children's hospitals and education centres. In addition, the PN could provide caregivers with materials to present to health-care providers that describe the navigation program services, which could help parents avoid repeating themselves. Our second recommendation would be that, when there is a transition between PNs, a meeting should be arranged between the ingoing and outgoing PN and the client to ease the disruption of the transition as was discussed in the limitations. Our final recommendation is that PNs consistently check in with stakeholders providing programs and services for the population they are serving, to ensure that the centre's repertoire of programs and services is up to date. Participant 5 was quoted above highlighting the back-and-forth relationship between caregivers and the PN.

\section{Conclusion}

All caregivers stated that they were satisfied with the services provided by NaviCare and found it to be a beneficial service. In particular, the PN was a primary source of emotional support for many parents, helping them through difficult situations. In general, this qualitative longitudinal descriptive case study demonstrates the benefit of patient navigation programs that focus on children with complex care needs and their families.

\section{References}

American Academy of Pediatrics Council on Children with Disabilities. (2005). Care coordination in the medical home: Integrating health and related systems of care for children with special health care needs. Pediatrics, 116(5), 12381244. https://doi.org/10.1542/peds.20052070

Bolger, N., Davis, A., \& Rafaeli, E. (2003). Diary methods: Capturing life as it is lived. Annual Review of Psychology, 54, 579616.

https://doi.org/10.1146/annurev.psyc h.54.101601.145030

Braun, V., \& Clarke, V. (2006). Using thematic analysis in psychology. Qualitative Research in Psychology, 3(2), 77-101. https://psycnet.apa.org/doi/10.1191/1 478088706qp063oa

Brenner, M., Kidston, C., Hilliard, C., Coyne, I., Eustace-Cook, J., Doyle, C., Begley, T., \& Barrett, M. J. (2018). Children's complex care needs: A systematic concept analysis of multidisciplinary language. European Journal of Pediatrics, 177(11), 1641-1652. https://doi.org/10.1007/s00431-0183216-9

Caicedo, C. (2014). Families with special needs children: Family health, functioning, and care burden. Journal of the American Psychiatric Nurses Association, 20(6), 398-407.

https://doi.org/10.1177\%2F10783903 14561326

Charlton, P., Azar, R., Luke, A., Doucet, S., Montelpare, W., Nagel, D., Hyndman, N., \& Thompson, K. (2017). Falling through the cracks: Barriers to accessing services for children with complex health conditions and their families in New Brunswick. Journal of New Brunswick Studies / Revue D'études Sur Le Nouveau-Brunswick, 8, 133-158. https://journals.lib.unb.ca/index.php/J NBS/article/view/25883

Colver, A. F., Merrick, H., Deverill, M., Le Couteur, A., Parr, J., Pearce, M. S., Rapley, T., Vale, L., Watson, R., \& McConachie, H. (2013). Study protocol: Longitudinal study of the transition of young people with complex health needs from child to adult health services. BMC Public Health, 13, Article 675. 
https://doi.org/10.1186/1471-245813-675

Doucet, S., Luke, A., Splane, J., \& Azar, R. (2019). Patient navigation as an approach to improve the integration of care: The case of NaviCare/SoinsNavi.

International Journal of Integrated Care, 19(4), Article 7. http://doi.org/10.5334/ijic.4648

Doucet, S., Nagel, D. A., Azar, R., Montelpare, W. J., Charlton, P., Hyndman, N., Luke, A., \& Stoddard, R. (2017). A mixed-methods Quick Strike research protocol to learn about children with complex health conditions and their families. International Journal of Qualitative Methods, 16(1). https://doi.org/10.1177\%2F16094069 17731426

Ellenwood, A. E., \& Jenkins, J. E. (2007). Unbalancing the effects of chronic illness: Non-traditional family therapy assessment and intervention approach. The American Journal of Family Therapy, 35(3), 265-277.

https://doi.org/10.1080/01926180600 968431

Fillion, L., Cook, S., Veillette, A. M., Aubin, M., de Serres, M., Rainville, F., Fitch, M., \& Doll, R. (2012). Professional navigation framework: Elaboration and validation in a Canadian context. Oncology Nursing Forum, 39(1), E58-69. https://doi.org/10.1188/12.onf.e58e69

Freeman, H. P., \& Rodriguez, R. L. (2011). History and principles of patient navigation. Cancer, 117(S15), 35393542. https://doi.org/10.1002/cncr.26262

Gotlib Conn, L., Hammond Mobilio, M., Rotstein, O. D., \& Blacker, S. (2016). Cancer patient experience with navigation service in an urban hospital setting: A qualitative study. European Journal of Cancer Care, 25(1), 132-140. https://doi.org/10.1111/ecc.12247

Kaufman, M., Pinzon, J., Canadian Paediatric Society, \& Adolescent Health
Committee. (2007). Transition to adult care for youth with special health care needs. Paediatrics \& Child Health, 12(9), 785-788.

https://doi.org/10.1093/pch/12.9.785

King, G., Williams, L., \& Hahn Goldberg, S. (2017). Family-oriented services in pediatric rehabilitation: A scoping review and framework to promote parent and family wellness. Child: Care, Health and Development, 43(3), 334347. https://doi.org/10.1111/cch.12435

Luke, A., Luck, K. E., \& Doucet, S. (2020). Experiences of caregivers as clients of a patient navigation program for children and youth with complex care needs: A qualitative descriptive study. International Journal of Integrated Care, 20(4), Article 10. http://doi.org/10.5334/ijic.5451

McMullen, L. (2013). Oncology nurse navigators and the continuum of cancer care. Seminars in Oncology Nursing, 29(2), 105-117. https://doi.org/10.1016/ j.soncn.2013.02.005

Paskett, E. D., Harrop, J. P., \& Wells, K. J. (2011). Patient navigation: An update on the state of the science. CA: A Cancer Journal for Clinicians, 61(4), 237-249. https://doi.org/10.3322/caac.20111

Valaitis, R. K., Carter, N., Lam, A., Nicholl, J., Feather, J., \& Cleghorn, L. (2017). Implementation and maintenance of patient navigation programs linking primary care with community-based health and social services: A scoping literature review. BMC Health Services Research, 17, Article 116. https://doi.org/ 10.1186/s12913-0172046-1

Weinhold, I., \& Gurtner, S. (2014). Understanding shortages of sufficient health care in rural areas. Health Policy (Amsterdam, Netherlands), 118(2), 201214. https://doi.org/10.1016/ j.healthpol.2014.07.018

Yin, R. K. (2014). Case study research: Design and methods (5th ed.). Sage Publication. 